\title{
A Vibrio harveyi insertional mutant in the cgtA (obg, yhbZ) gene, whose homologues are present in diverse organisms ranging from bacteria to humans and are essential genes in many bacterial species
}

\author{
Agata Czyż, ${ }^{1}$ Ryszard Zielke, ${ }^{2}$ Grażyna Konopa ${ }^{2}$ and Grzegorz Węgrzynn ${ }^{2,3}$
}

Author for correspondence: Grzegorz Węgrzyn. Tel: +48 58346 3014. Fax: + 48583010072.
e-mail: wegrzyn@ biotech.univ.gda.pl

1 Laboratory of Molecular Biology (affiliated with the University of Gdańsk), Institute of Biochemistry and Biophysics, Polish Academy of Sciences, Kładki 24, 80-822 Gdańsk, Poland

2 Department of Molecular Biology, University of Gdańsk, Kladki 24, 80-822 Gdańsk, Poland

3 Marine Biology Center, Polish Academy of Sciences, Św. Wojciecha 5, 81-347 Gdynia, Poland
The cgtA gene product is a member of the subfamily of small GTP-binding proteins that have been identified in diverse organisms ranging from bacteria to humans. In bacteria that sporulate or display another special developmental programme, this gene (referred to as cgtA, obg or yhbZ) appears to be involved in the regulation of these processes. However, this gene has also been found to be essential in all bacterial species investigated to date, although its role in bacteria that do not sporulate and do not undergo a specific development remains unknown. Here the authors characterize a Vibrio harveyi mutant bearing a transposon insertion into the cgtA gene. This mutant reveals a multiple phenotype: it grows more slowly than the wild-type strain in a rich medium; its growth is completely inhibited in minimal media; its survival in $3 \% \mathrm{NaCl}$ is dramatically reduced; it is very sensitive to UV irradiation; it is more susceptible to mutation upon treatment with different mutagens; its luminescence is decreased; its quorum-sensing regulation is less effective than in the wild-type strain; and the elongated shape of the mutant cells may suggest problems with the regulation of cell division and/or DNA replication. These defects in diverse cellular processes found in the insertional cgtA mutant of $V$. harveyi indicate that in a bacterium that does not sporulate and does not display other special development programmes, the CgtA protein is involved in the regulation of many crucial biochemical reactions, possibly at the stage of signal transduction.

Keywords: $\operatorname{cgtA}$ gene, GTP-binding protein, bioluminescence, signal transduction

\section{INTRODUCTION}

GTP-binding proteins play crucial roles in the regulation of fundamental processes in cells of all living organisms. In fact, small monomeric GTP-binding proteins have been found in every organism examined thus far. In eukaryotic cells, these proteins are involved in a number of essential processes, such as signal transduction, protein synthesis and translocation or cell cycle regulation (for a review see, for example, Sprang, 1997). However, perhaps surprisingly, relatively little infor-

Abbreviation: DAPI, 4',6-diamidino-2-phenylindole.

The GenBank accession number for the sequence reported in this paper is AF247677. mation is currently available about the roles of GTPbinding proteins in prokaryotes.

The best-studied prokaryotic GTP-binding protein is Era (for 'Escherichia coli Ras-like protein'). This protein is essential for bacterial growth, and mutants in the era gene have pleiotropic phenotypes, including alterations in the regulation of carbon metabolism, the stringent response, and cell division (Lerner \& Inouye, 1991; Britton et al., 1997, 1998). Recent studies have demonstrated that Era bears an RNA-binding motif (Chen et al., 1999) and binds to the 30S ribosomal subunit (Sayed et al., 1999).

Apart from Era, a subfamily of small GTP-binding proteins was discovered recently in bacteria. Interestingly, members of this subfamily have homo- 
logues in diverse organisms ranging from bacteria to humans (Okamoto \& Ochi, 1998). This subfamily is $\mathrm{Obg} / \mathrm{Gtp} 1$, and examples of bacterial members of it are Obg from Bacillus subtilis, Streptomyces griseus and $S$. coelicolor, CgtA from Caulobacter crescentus, and YhbZ from Escherichia coli and Haemophilus influenzae. Among this group, the B. subtilis obg gene product is the best-studied protein. Genetic studies led to proposals that Obg may regulate initiation of sporulation (Trach \& Hoch, 1989; Vidwans et al., 1995), may be involved in the control of DNA replication (Kok et al., 1994), and is necessary for stress-dependent activation of transcription factor $\sigma^{\mathrm{B}}$ (Scott \& Haldenwang, 1999). It was proposed that Obg can function by sensing the intracellular GTP level (Kok et al., 1994) and may be required to stimulate the activity of the phosphorelay system (Vidwans et al., 1995). Therefore, it is likely that Obg may be involved in signal transduction.

Functions of $o b g$ and $c g t A$ genes have been investigated to date in bacteria that display a development programme, i.e. in B. subtilis, S. griseus, S. coelicolor and C. crescentus. However, members of the Obg/Gtp1 subfamily, with a high homology to Obg and CgtA, were found to be essential also in bacteria that do not sporulate and do not differentiate. For example, the $y h b Z$ gene of E. coli was demonstrated to be essential (Arigoni et al., 1998), whereas its role in this bacterium is unknown. Investigation of the role of an essential gene is complicated by the fact that it is not possible to obtain viable null mutants. In the case of $B$. subtilis, apart from a temperature-sensitive obg mutant (Kok et al., 1994), a strain in which the only functional obg gene copy is under control of an IPTG-inducible promoter has been constructed (Vidwans et al., 1995). This strain is viable only in the presence of the inducer in the medium. Without IPTG-induced transcription of $o b g$, the levels of the Obg protein were depleted during the cell growth; however, cells appeared to grow normally for several generations before there was any significant change relative to the same strain grown in the presence of IPTG (Vidwans et al., 1995). This allowed investigation of the influence of $o b g$ gene function on sporulation, but such a phenotype would cause difficulties in studies on many other processes potentially affected by this gene function. Moreover, using an IPTG-inducible transcription of a given gene, it is difficult to obtain a level of the gene product comparable to that found in the wild-type strain.

Vibrio harveyi is a Gram-negative, free-living, luminescent marine bacterium. Here we describe a $V$. harveyi mutant which bears a transposon insertion in the cgt $A$ $(o b g, y b b Z)$ gene. To our knowledge, this is the first reported viable bacterial mutant in which a gene belonging to the $\mathrm{Obg} / \mathrm{Gtp} 1$ subfamily has been disrupted by insertion.

\section{METHODS}

Bacterial strains and plasmids. $V$. harveyi wild-type strain BB7 (Belas et al., 1982) and its cgtA:: Tn5TpMCS derivative,
BB7X (Czyż et al., 2000a), were used. Plasmid pSup Tn5pMCS (MacKenzie et al., 1995) bears a Tn5-derived transposon carrying a trimethoprim-resistance gene. Plasmid pUC19 has been described previously (Yanisch-Perron et al., 1985). All genetic engineering procedures used for plasmid construction described below were performed according to Sambrook et al. (1989). For construction of plasmid pAC1, bearing a chromosomal DNA fragment flanking the site of transposon insertion, a procedure described by MacKenzie et al. (1995) was employed. Briefly, chromosomal DNA isolated from $V$. harveyi BB7X was digested with EcoRI and mixed with EcoRI-digested pUC19. Following ligation, the transformation of E. coli MC1061 (Meissner et al., 1987) was performed using selection for resistance to both ampicillin and trimethoprim.

Culture media. Luria-Bertani (LB) medium (used for E. coli cultivation) and BOSS medium (a rich medium used for $V$. harveyi cultivation) have already been described by Sambrook et al. (1989) and Klein et al. (1998), respectively. Minimal medium 3 (Węgrzyn \& Taylor, 1992) was used, but the concentration of $\mathrm{NaCl}$ was $3 \%$, and the following carbon sources were employed: $1 \%$ glucose plus $1 \%$ Casamino acids; $1 \%$ glucose; $1 \%(\mathrm{v} / \mathrm{v})$ glycerol $; 1 \%$ sodium succinate; or $1 \%$ sodium acetate. If not indicated, V. harveyi strains were cultivated at $30^{\circ} \mathrm{C}$

DNA sequencing. A fragment of plasmid pAC1 (bearing the $V$. harveyi BB7X DNA region flanking the site of transposon insertion) was sequenced automatically using a Perkin-Elmer ABI 310 sequencer. The ABI Prism BigDye Terminator Cycle Sequencing Reaction Kit with Ampli Taq DNA Polymerase FS was employed. The primers used for sequencing reactions were as follows: primer TN, 5'-TTC AGG ACG CTA CTT GTG TA-3'; and primer pUCR, 5'-AGC GAA TAA CAA TTT CAC ACA GG-3'.

Electron microscopy. V. harveyi cells were examined by electron microscopy using two techniques: negative staining with phosphotungstic acid (PTA), and ultrathin sectioning. For the first technique, cells were prepared by negative staining with $0 \cdot 2-1 \%$ PTA (neutralized with $\mathrm{KOH}$ to $\mathrm{pH} 7 \cdot 5$ ) on carbon-coated copper grids according to Quintarelli et al. (1971). Briefly, the grid was placed on the top of a drop of bacterial culture and left for $30 \mathrm{~s}$, and then transferred to the top of a drop of the PTA solution for $30 \mathrm{~s}$. Excess stain was removed with filter paper and the grid was dried at room temperature. For the second technique, the basic methods used to fix and embed cells for thin sectioning were according to Spurr (1969). Sections were stained with saturated uranyl acetate dissolved in $50 \%$ ethanol, and then with lead citrate for $2 \mathrm{~min}$ at room temperature. The grid was dried at room temperature. In both techniques, the grids were examined and photographed using a Philips CM 100 electron microscope operating at $60 \mathrm{kV}$.

Light microscopy. V. harveyi cells were examined using differential interference contrast under a Nikon Eclipse E800 microscope. Staining with 4',6-diamidino-2-phenylindole (DAPI) was carried out essentially as described previously by Hause et al. (1993). Briefly, culture samples were mixed with DAPI solution $\left(25 \mu \mathrm{g} \mathrm{ml}^{-1}\right)$ and left in the dark for $20 \mathrm{~min}$. For staining with ethidium bromide, culture samples were mixed with the dye solution $\left(100 \mu \mathrm{g} \mathrm{ml}^{-1}\right)$ and left in the dark for $20 \mathrm{~min}$. In both staining procedures, before examination under a microscope, the stained cells were placed on a $0.5 \%$ agar layer on a microscope slide and covered with a coverslip.

Southern blotting. Southern-blotting analysis was performed according to Sambrook et al. (1989) using the Random Primer Fluorescein Labelling Kit with Antifluorescein-AP (NEN Life 


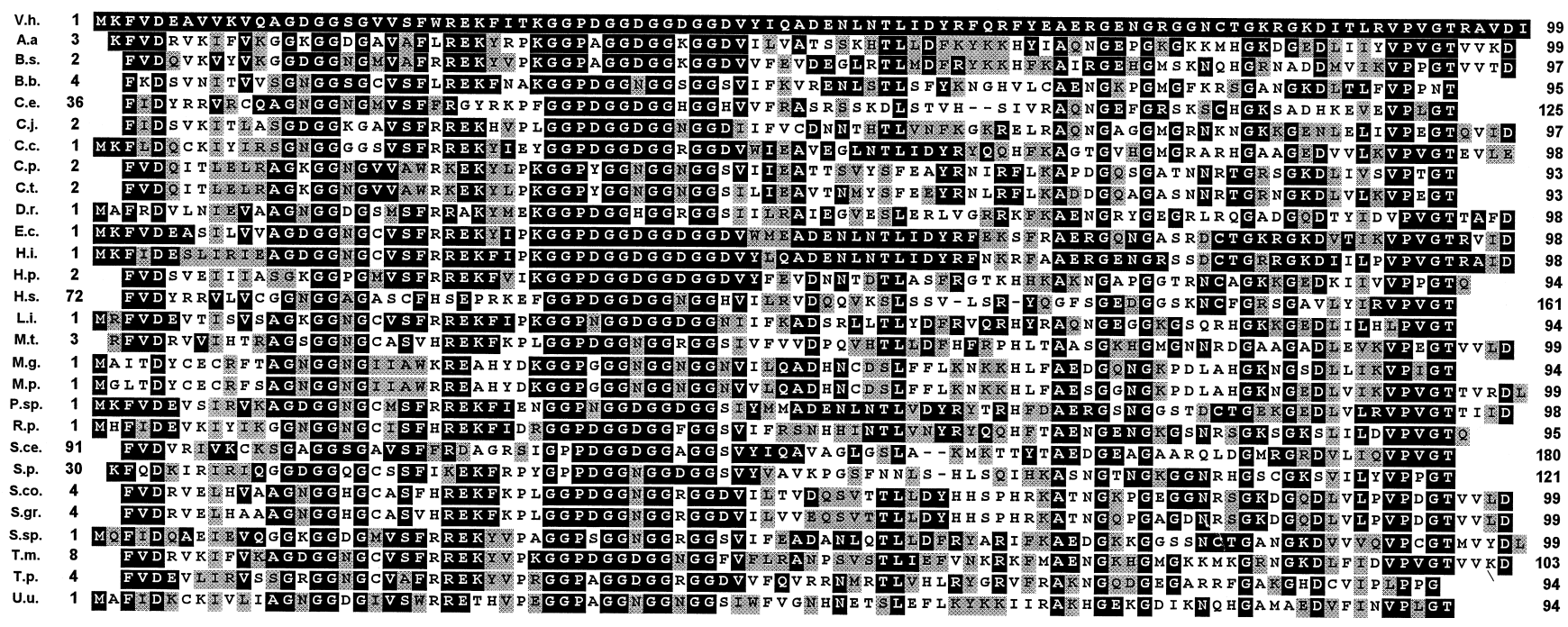

Fig. 1. Homology of the $\mathrm{N}$-terminal (99 amino acids) part of the $V$. harveyi CgtA protein (the sequence was deduced on the basis of the partial cgtA gene sequence) with corresponding regions of certain proteins belonging to the Obg/Gtp1 subfamily from diverse organisms. The wu-blastp software was used for comparison of protein sequences taken from the SWALL database. Identity (white letters on black background) or similarity (black letters on grey background) of particular amino acid residues in the given proteins from different organisms to the $\mathrm{N}$-terminal part of the $V$. harveyi CgtA protein (first line) is shown (black letters on white background indicate no identity and similarity to the $V$. harveyi CgtA sequence). The first and the last residues of the compared regions of proteins are indicated at the beginning and at the end of the respective sequence. The abbreviations of the names of species are as follows (the symbols in parentheses represent SWALL database accession numbers): V.h., Vibrio harveyi; A.a., Aquifex aeolicus (O67849); B.s., Bacillus subtilis (P20964); B.b.; Borrelia burgdorferi (051722); C.e., Caenorhabditis elegans (O45691); C.j., Campylobacter jejuni (CAB72580); C.C., Caulobacter crescentus (O30861); C.p., Chlamydia pneumoniae (Q9Z808); C.t., Chlamydia trachomatis (O84423); D.r., Deinococcus radiodurans (AAF09676); E.C., Escherichia coli (P42641); H.i., Haemophilus influenzae (P44915); H.p., Helicobacter pylori (Q9ZMD3); H.s., Homo sapiens (BAA91783); L.i., Lawsonia intracellularis (O87884); M.t., Mycobacterium tuberculosis (P71909); M.g., Mycoplasma genitalium (P47624); M.p., Mycoplasma pneumoniae (P75215); P.sp., Pseudomonas sp. BG33R (AAD47628); R.p., Rickettsia prowazekii (Q9ZCB6); S.ce., Saccharomyces cerevisiae (P38860); S.p., Schizosaccharomyces pombe (CAB55178); S.co., Streptomyces coelicolor (P95722); S.gr., Streptomyces griseus (P95758); S.sp., Synechocystis sp. PCC6803 (P72931); T.m., Thermotoga maritima (Q9WXV3); T.p., Treponema pallidum (O83724); U.u., Ureoplasma urealyticum (AAF30873).

Science Products) for probe labelling and detection. The templates for preparation of probes were the EcoRI-PvuII fragment of plasmid pSupTn5pMCS (encompassing a trimethoprim-resistance gene from the transposon) and the EcoRV-BglII fragment of plasmid pAC1 (encompassing a part of the $V$. harveyi cgtA gene).

Measurement of bioluminescence. Bacteria were grown to high cell density in BOSS medium. Then the cultures were diluted 10000-fold in the fresh medium and cultivation was continued. Samples were withdrawn at intervals; each sample was centrifuged $(2000 \mathrm{~g}, 5 \mathrm{~min}$ ) and the pellet was resuspended in an equal volume of $3 \% \mathrm{NaCl}$. The number of bacteria was determined by plating and luminescence was monitored in a scintillation counter using chemiluminescence mode as described previously (Bassler et al., 1994). The relative light units were calculated as counts $\mathrm{min}^{-1} \mathrm{ml}^{-1}$ per cell.

Survival of cells under starvation conditions. Bacterial cultures growing in BOSS medium were centrifuged; pellets were washed with $3 \% \mathrm{NaCl}$ and resuspended in an equal volume of this solution. The suspensions were incubated at $30^{\circ} \mathrm{C}$; samples were withdrawn at indicated times and the number of living cells was determined by plating on BOSS plates.

UV sensitivity assay. Bacteria were cultivated in BOSS medium, centrifuged and resuspended in $3 \% \mathrm{NaCl}$. Then $1 \times 10^{8}$ cells were irradiated with different UV doses followed by incubation in BOSS medium in the dark for $2 \mathrm{~h}$ and titration on BOSS plates (the plates were incubated overnight in the dark).

Measurement of RNA synthesis. The experiments were performed as described previously by Wegrzyn et al. (1991). Briefly, bacteria were grown in BOSS medium to midexponential phase; $\left[{ }^{3} \mathrm{H}\right]$ uridine was added to $2 \mu \mathrm{Ci} \mathrm{ml} \mathrm{m}^{-1}$ $\left(74 \mathrm{kBq} \mathrm{ml}^{-1}\right)$ and incubation was continued. At indicated times samples $(50 \mu \mathrm{l}$ each) were withdrawn to paper filters and transferred immediately to ice-cold $10 \%$ trichloroacetic acid (TCA). After $5 \mathrm{~min}$ incubation in an ice bath, the filters were transferred to ice-cold $5 \%$ TCA for $5 \mathrm{~min}$, and then washed twice in $96 \%$ ethanol. After drying at room temperature, the radioactivity of the samples was measured in a scintillation counter. To induce the stringent response, serine hydroxamate was added to the culture up to $1 \mathrm{mg} \mathrm{ml}^{-1}$.

\section{RESULTS}

\section{Determination and analysis of a partial nucleotide sequence of the $V$. harveyi cgtA gene and a deduced amino acid sequence of its product}

We have reported previously the isolation of a number of random insertional $V$. harveyi mutants obtained after transposon mutagenesis (Czyż et al., 2000a, b). Here we 

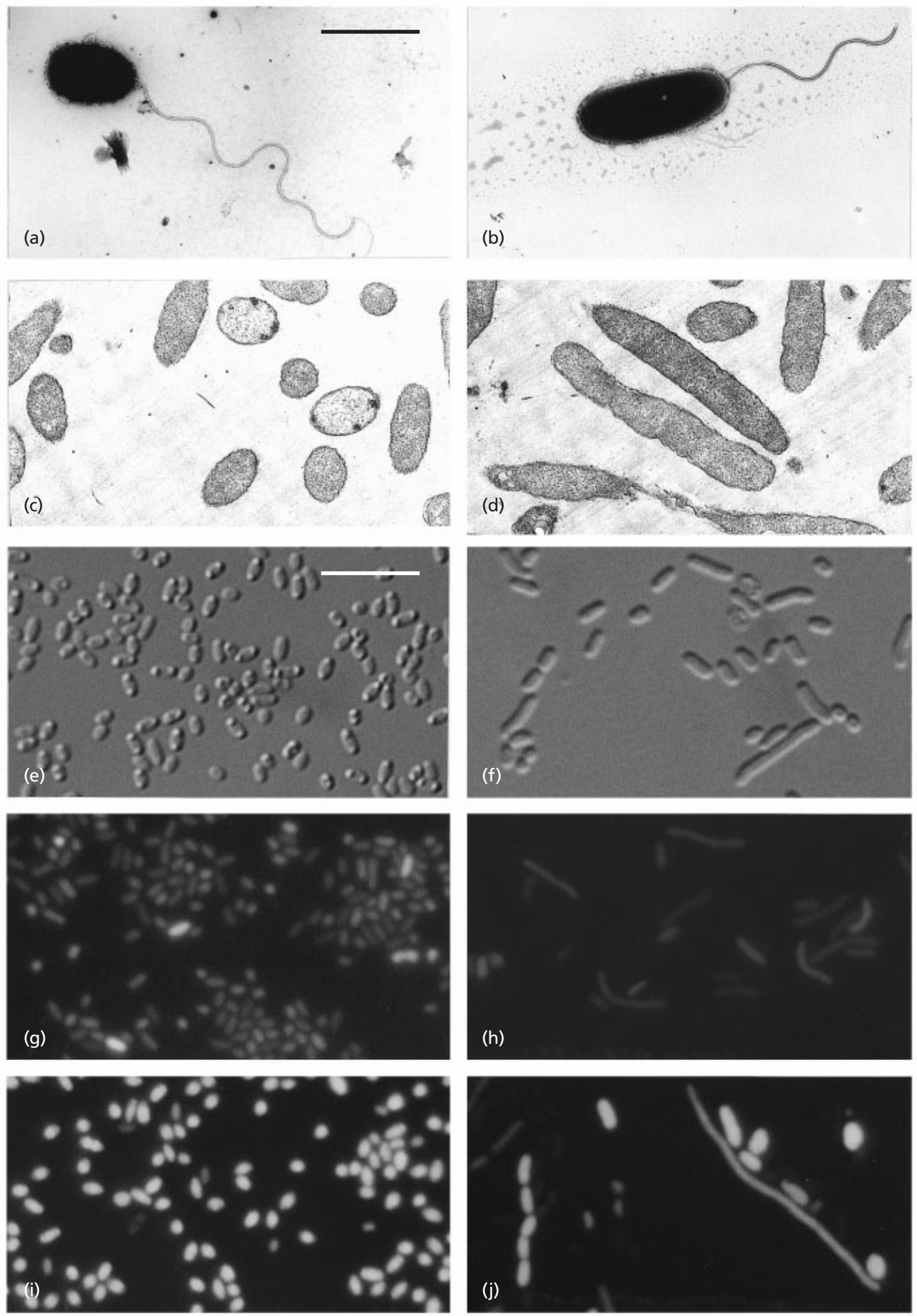

Fig. 2. Morphology of cells of $V$. harveyi wild-type strain $(a, c, e, g, i)$ and the cgtA::Tn5TpMSC mutant $(b, d, f, h$, j). The cells were photographed under an electron microscope $(a, b, c, d)$ or under a light microscope using differential interference contrast $(e, f, g, h, i, j)$. The specific treatments of the cells were as follows: staining with PTA $(a, b)$, ultrathin sectioning $(c, d)$, staining with DAPI $(g, h)$, and staining with ethidium bromide $(i, j)$. The bar in $(a)$ represents $2 \mu m$ for the electron micrographs $(a-d)$; the bar in (e) represents $7.5 \mu \mathrm{m}$ for the other photographs $(e-j)$. 
aimed to characterize one of these mutants, strain BB7X. To determine which gene had been disrupted by a transposon insertion in this strain, $V$. harveyi $\mathrm{BB} 7 \mathrm{X}$ total DNA was digested with EcoRI and the fragment bearing a part of the transposon and flanking DNA sequence was subcloned into pUC19, using selection for ampicillin (an ampicillin-resistance gene is present in the vector) and trimethoprim (a trimethoprim-resistance gene is present in the fragment of the transposon). Then, the DNA region adjacent to the transposon was sequenced using primers complementary to the end of the transposon and to the pUC19 vector. Thus, both DNA strands of this region have been sequenced. The sequence obtained (GenBank accession number AF247677) revealed that the transposon had been inserted into a gene that has a high homology to the $y b b Z$ gene of E. coli and H. influenzae (data not shown). The insertion occurred after 99th codon of the gene (data not shown).

Analysis of the putative amino acid sequence of the Nterminal 99 amino acids of the investigated gene product indicated a high percentage of identity/similarity to small GTP-binding proteins from diverse organisms ranging from bacteria to humans (Fig. 1). This analysis indicated that the gene product belongs to the Obg/Gtp1 subfamily. The best-investigated bacterial members of this subfamily of proteins are Obg (for 'SpoOBassociated GTP-binding protein') from B. subtilis, S. griseus and S. coelicolor and CgtA (for 'Caulobacter GTP-binding protein') from C. crescentus, although our knowledge of the roles of these proteins and the corresponding genes is very limited. To avoid further confusion in the nomenclature of homologous proteins, we propose to keep the name CgtA for the $V$. harveyi member of the Obg/Gtp1 subfamily (however, the name CgtA would be for 'common GTP-binding protein' rather than for 'Caulobacter GTP-binding protein').

It was intriguing that $\operatorname{cgt} A(y b b Z, o b g)$-null mutants of other bacteria investigated to date were not viable (Arigoni et al., 1998), whereas we have isolated a viable $\operatorname{cgt} A$ insertional mutant of $V$. harveyi. Therefore, we aimed to characterize this mutant in more detail, as such studies could provide important information about the role and function of the $\operatorname{cgt} A$ gene product and homologous proteins. To verify whether the transposon is located in the single locus in $V$. harveyi strain BB7X, and thus whether all observed phenotypes result solely from disruption of the cgtA gene, we performed Southern-blotting analysis. Total DNA isolated from strain BB7X was digested with EcoRI, subjected to agarose gel electrophoresis, transferred to a nylon membrane and hybridized to a fluorescein-labelled probe specific to a fragment of the transposon. If the transposon was located in the single locus we should observe one specific band, whereas location of the transposon in two or more regions of the chromosome should result in appearance of multiple bands after the Southern blotting. Indeed, we observed a single band (data not shown), which indicates that the transposon is located in a unique site in the chromosome of strain
$\mathrm{BB} 7 \mathrm{X}$, and the subcloning and sequence analysis described above determined that this site is the $\operatorname{cgt} A$ gene.

One could argue that $V$. harveyi might contain a homologue of the cgtA gene whose expression could partially suppress effects of $c g t A$ dysfunction, making the insertional mutant described in this report viable, in contrast to other bacteria devoid of such a homologue. To test such a possibility, we performed Southernblotting analysis using EcoRI-digested chromosomal DNA of the wild-type V. harveyi (strain BB7). Following agarose gel electrophoresis, transfer of DNA to nylon membrane, and hybridization with a fluorescein-labelled probe prepared using the evolutionarily conserved $5^{\prime}$ region of the $\operatorname{cgt} A$ gene of $V$. harveyi as a template (the EcoRV-BglII fragment of plasmid pAC1), we observed a single band (data not shown). This makes the hypothesis of the presence of a homologue of the $\operatorname{cgt} A$ gene in $V$. harveyi chromosome less likely.

\section{Morphology of colonies and cells of the cgtA mutant}

The cgtA::Tn5TpMCS mutant formed considerably smaller colonies on plates with nutrient agar medium relative to the otherwise isogenic wild-type strain (data not shown). Moreover, the colonies of the mutant were less luminescent than those of the wild-type $V$. harveyi (data not shown).

The morphology of mutant cells also differed from that of the wild-type bacteria. This was revealed using electron microscopy and light microscopy with interference contrast and after staining with ethidium bromide and DAPI (Fig. 2). Generally, the cgtA mutant cells were significantly longer than normal $V$. harveyi cells, i.e. they had a tendency to form filaments. Such a morphology of cells may suggest problems with cell division and/or regulation of DNA replication. The long $V$. harveyi cgtA:: Tn5TpMCS cells stained quite uniformly with the DNA-binding dyes, suggesting that there are problems with regulation of DNA replication rather than with segregation of genomes to daughter cells. Frequent formation of chains of cells (Fig. 2j) supports the suggestion of problems with cell division in the mutant.

\section{Quorum sensing by the cgtA mutant}

Luminescence in $V$. harveyi is regulated by the quorumsensing mechanism (for reviews see Bassler \& Silverman, 1995; Swift et al., 1998). Due to this regulation, the luminescence is induced only when bacteria are at high cell density because expression of the appropriate genes is dependent on the presence in the medium of an autoinducer. This autoinducer is produced by $V$. harvey $i$ cells and its synthesis is regulated according to a positive feedback mechanism. Therefore, only cells growing at high cell density can produce a sufficient amount of the inducer.

Wild-type $V$. harveyi and the $\operatorname{cgt} A$ mutant were grown to high cell density (to allow efficient luminescence), and then the cultures were diluted 10000-fold in fresh 


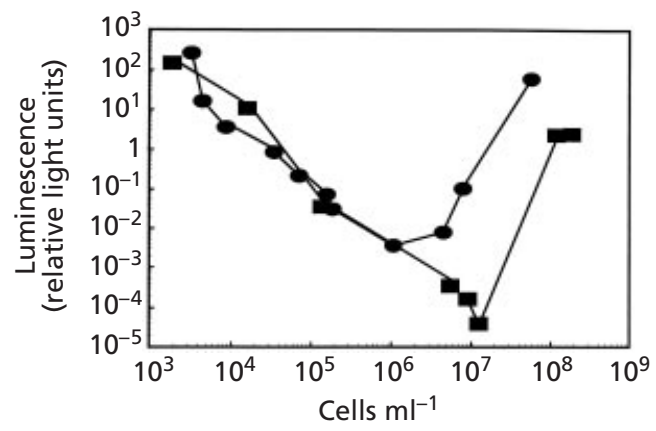

Fig. 3. Luminescence of $V$. harveyi wild-type cells (circles) and the cgtA::Tn5TpMSC mutant (squares) at different cell densities. Cultures growing exponentially in BOSS medium were diluted 10000 -fold at time 0 , and luminescence of samples withdrawn from the cultures at the indicated cell densities was measured. The relative light units were calculated as counts $\mathrm{min}^{-1} \mathrm{ml}^{-1}$ per cell. The values presented are means from three experiments. In all cases, the standard deviation was below $10 \%$ of the mean.

medium. Cultivation was continued, and luminescence and cell density were measured at intervals. In the culture of the wild-type cells, the luminescence decreased after dilution and then became more effective due to the increase in the cell density of the culture (Fig. 3). The same phenomenon was observed in the culture of the $\operatorname{cgt} A$ mutant; however a significantly higher cell density had to be achieved to stimulate luminescence (Fig. 3). These results indicate that the quorum-sensing regulation may be less efficient in the $\operatorname{cgt} A$ mutant in comparison to the wild-type strain.

\section{Sensitivity of the cgtA mutant to mutagenic agents}

$V$. harveyi strains, including BB7 and BB7X, have previously been proposed as bioindicators of mutagenic pollution of the environment (Czyż et al., 2000a). Higher sensitivity of strain BB7X (characterized in this report as the $\operatorname{cgtA}:: \operatorname{Tn} 5 \mathrm{TpMSC}$ mutant) to UV irradiation in a preliminary plate test has been reported (Czyż et al., 2000a). Here the survival of bacteria after irradiation with various UV doses was measured in more detail. We found that the cgtA mutant was significantly more sensitive to UV irradiation than the wild-type (Fig. 4). Higher mutability of strain BB7X in response to various mutagens, relative to the isogenic wild-type strain BB7, was reported previously (Czyż et al., 2000a). We conclude that DNA repair is impaired in the $\operatorname{cgt} A$ mutant.

\section{Inhibition of growth of the cgtA mutant in minimal media}

As mentioned above, the cgtA mutant formed smaller colonies on nutrient agar plates than the wild-type strain. We also observed slower growth of the $\operatorname{cgt} A$ mutant in the rich liquid medium relative to the wildtype strain (data not shown). Moreover, we found that

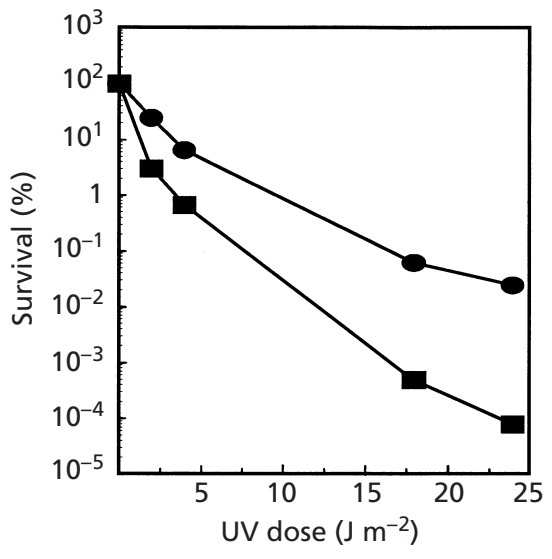

Fig. 4. Sensitivity to UV irradiation of $V$. harveyi wild-type cells (circles) and the cgtA::Tn5TpMSC mutant (squares). Bacteria from exponentially growing cultures were UV irradiated (with the indicated doses) and the number of survivors was determined by plating.

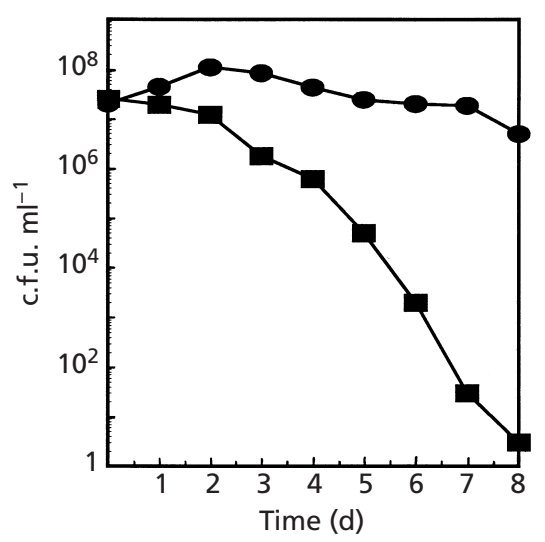

Fig. 5. Survival of $V$. harveyi wild-type cells (circles) and the cgtA::Tn5TpMSC mutant (squares) in $3 \% \mathrm{NaCl}$. Bacterial cultures growing in BOSS medium were centrifuged; the pellets were washed with $3 \% \mathrm{NaCl}$ and resuspended in equal volumes of this solution. The bacterial suspensions were incubated at $30^{\circ} \mathrm{C}$ and samples for titration (on BOSS plates) were withdrawn at the indicated times.

in contrast to wild-type bacteria the $\operatorname{cgt} A$ mutant was not able to grow in minimal media with various carbon sources (see Methods) (data not shown).

\section{Survival of the cgtA mutant during starvation}

Because of the inhibition of growth of the $\operatorname{cgt} A$ mutant in minimal media, we measured its viability under starvation conditions. Bacteria were incubated in 3\% $\mathrm{NaCl}$ (this salt concentration is optimal for $V$. harveyi) for several days and the number of survivors was measured by plating of the cell suspensions. Viability of the $\operatorname{cgt} A$ mutant cells was dramatically decreased under these conditions relative to the wild-type (Fig. 5). 


\section{The insertion in the cgtA gene does not influence the stringent response or the heat-shock response}

Since the cgtA mutant revealed defects in its growth in minimal media, and its viability in $3 \% \mathrm{NaCl}$ was significantly impaired, we asked whether the stringent response, a bacterial response to amino acid and carbon source starvation, is affected by $\operatorname{cgt} A$ dysfunction. The first effect of the stringent response is production of a specific nucleotide, guanosine tetraphosphate (ppGpp), which by interacting with RNA polymerase inhibits synthesis of stable RNAs (for a review see Cashel et al., 1996). We measured RNA synthesis in cells growing in normal conditions and after induction of the stringent response, achieved by addition of serine hydroxamate to the medium. However, we did not observe any significant differences between the $\operatorname{cgt} A$ mutant and the wild-type strain. In both strains addition of serine hydroxamate resulted in rapid inhibition of RNA synthesis (data not shown).

We also investigated the response of the $\operatorname{cgt} A$ mutant to another stress agent, increase in temperature. It was demonstrated previously that $V$. harveyi shows a typical heat-shock response, although at temperatures somewhat lower than E. coli (Klein et al., 1995). However, we failed to detect any difference in the heat-shock response between the $\operatorname{cgtA}$ mutant and the wild-type strain (data not shown).

\section{DISCUSSION}

In this study we have characterized a $V$. harveyi strain in which the cgtA gene, an obg homologue, has been disrupted by transposon insertion. To our knowledge, this strain is the first example of an insertional mutant in genes encoding bacterial members of the Obg/Gtp1 proteins. Although it seems likely that the transposon insertion inactivated the $\operatorname{cgt} A$ gene, we cannot be sure that strain BB7X is a true null mutant. The insertion occurred after the 99th codon of the cgtA gene; thus it is possible that synthesis of the N-terminal part of CgtA is sufficient to retain some functions of this protein. Another possibility could be that the phenotypes of the mutant do not result directly from the cgtA gene dysfunction but are polar effects of the transposon insertion. This might occur if any important genes were located downstream of cgtA in the same operon. However, analysis of the structure of the E. coli operon containing the $y h b Z$ gene (a cgtA homologue) revealed that it consists of four genes: $r p l U, r p m A, y b b E$ and $y b b Z$, where the $\operatorname{cgt} A$ homologue is the most distal gene. Considering the high homology between E. coli $y b b Z$ and $V$. harveryi cgtA genes, and the close relation of these two bacterial species, it is likely that the organization of a homologous operon in $V$. harveyi resembles that of $E$. coli. If this is true, no polar effects of the $\operatorname{cgt} A:: \operatorname{Tn} 5 \mathrm{TpMCS}$ mutation should occur.

The $V$. harveyi cgt A mutant revealed multiple defects in several cellular processes. Formation of cell filaments (Fig. 2) may suggest problems with regulation of cell division and/or DNA replication at least under certain conditions. In fact, it was proposed previously that the Obg protein of B. subtilis may be involved in the regulation of DNA replication (Kok et al., 1994). The same $B$. subtilis protein was recently demonstrated to be involved in activation of the $\sigma^{\mathrm{B}}$ factor (Scott \& Haldenwang, 1999). This factor is the general stressresponse $\sigma$ subunit of RNA polymerase that is activated when intracellular ATP levels fall or the bacterium experiences environmental stress. Activity of $\sigma^{\mathrm{B}}$ is regulated by a set of kinases and phosphatases, called the Rsb proteins, which catalyse the release of $\sigma^{\mathrm{B}}$ from an anti- $\sigma$ factor. It was suggested that Obg may interact with one or more $\sigma^{\mathrm{B}}$ regulators (Scott \& Haldenwang, 1999). The cgt A mutant of $V$. harveyi is not able to grow in minimal media and its viability in $3 \% \mathrm{NaCl}$ is dramatically decreased (Fig. 5), suggesting that induction of expression of certain genes that are normally active under conditions of starvation and energy deprivation may be impaired. Therefore, one may speculate that activation of an appropriate $\sigma$ factor (e.g. a homologue of $E$. coli $\sigma^{\mathrm{S}}$ ) could be dependent on the $\operatorname{cgt} A$ gene function, similarly to the obg-dependent activation of $B$. subtilis $\sigma^{\mathrm{B}}$. However, it is worth noting that in the case of the B. subtilis obg gene dysfunction the $\sigma^{\mathrm{B}}$ factor was no longer activated in response to environmental stress, but it retained the ability to be activated by the ATP-responsive pathway (Scott \& Haldenwang, 1999). Thus, we should also consider that the mechanism of $\operatorname{cgt} A$-dependent $V$. harveyi growth in minimal media and survival under starvation conditions might be significantly different from that of $B$. subtilis.

Colonies of the cgtA mutant of $V$. harveyi were less luminescent than those of the wild-type. However, calculation of the relative luminescence per single bacterium in a liquid culture of high cell density revealed significantly smaller differences (Fig. 3). This could suggest defects in the quorum-sensing regulation rather than in the luminescence reaction itself. Indeed, the $\operatorname{cgt} A$ mutant required significantly higher cell density for efficient quorum-sensing-dependent induction of luminescence relative to the wild-type strain (Fig. 3). The quorum-sensing regulation in $V$. harveyi requires activation of kinases and phosphatases (Freeman \& Bassler, 1999a, b; Freeman et al., 2000). Therefore, it seems likely that the $c g t A$ gene product may be involved in the process of signal transduction during the quorumsensing regulation.

Currently it is difficult to explain the mechanism of decreased efficiency of DNA repair in the $\operatorname{cgt} A$ mutant (Fig. 4, and Czyż et al., 2000a). One possibility is that decreased efficiency of light emission in this mutant results in less efficient photoreactivation, since it was proposed recently that bioluminescence may stimulate photolyase activity (Czyż et al., 2000b). On the other hand, CgtA might be involved in the regulation of expression of genes encoding DNA repair proteins.

In conclusion, the cgt $A:: \operatorname{Tn} 5$ TpMCS mutant of $V$. harveyi reveals multiple defects in important cellular processes, although it is still viable in rich media, in contrast to null mutants in the homologous genes of 
other bacteria investigated to date, which are not viable. Most, if not all, of the processes affected by the cgtA dysfunction in $V$. harveyi and reported in this article involve regulation of gene expression in response to various environmental signals. The $V$. harveyi cgt $A$ gene product is a potential GTP-binding protein as it has a high homology to such proteins identified in many other organisms (Fig. 1). Therefore, it seems likely that $V$. harveyi CgtA protein is involved in signal-transduction processes. According to this speculation, bacterial responses to environmental stresses that apparently do not require protein phosphorylation/dephosphorylation-mediated signal transduction, e.g. the stringent response and the heat-shock response, appear to be normal in the $\operatorname{cgt} A$ mutant of $V$. harveyi.

\section{ACKNOWLEDGEMENTS}

We thank Jerzy Bohdanowicz and Beata Lewandowska for their help during light microscopy studies. We are grateful to Joanna Potrykus and Katarzyna Potrykus for critical reading of the manuscript. This work was supported by University of Gdańsk (grant BW/1190-5-0120-0 to A.C.). G.W. acknowledges also a financial support from the Foundation for Polish Science (subsydium 14/2000).

\section{REFERENCES}

Arigoni, F., Talabot, F., Peitsch, M., Edgerton, M. D., Meldrum, E., Allet, E., Fish, R., Jamotte, T., Curchod, M.-L. \& Loferer, H. (1998). A genome-based approach for the identification of essential bacterial genes. Nat Biotechnol 16, 851-856.

Bassler, B. L. \& Silverman, M. R. (1995). Intercellular communication in marine Vibrio: density-dependent regulation of the expression of bioluminescence. In Two-component Signal Transduction, pp. 431-445. Edited by J. A. Hoch \& T. J. Silhavy. Washington, DC: American Society for Microbiology.

Bassler, B. L., Wright, M. \& Silverman, M. R. (1994). Multiple signalling systems controlling expression of luminescence in Vibrio harveyi: sequence and function of genes encoding a second sensory pathway. Mol Microbiol 13, 273-286.

Belas, R., Mileham, A., Cohn, D., Hilmen, M., Simon, M. \& Silverman, M. (1982). Bacterial luminescence: isolation and expression of the luciferase genes from Vibrio harveyi. Science 218, 791-793.

Britton, R. A., Powell, B. S., Court, D. L. \& Lupski, J. R. (1997). Characterization of mutations affecting the Escherichia coli essential GTPase Era that suppress two temperature-sensitive dna $G$ alleles. J Bacteriol 179, 4547-4582.

Britton, R. A., Powell, B. S., Dasgupta, S., Sun, Q., Margolin, W., Lupski, J. R. \& Court, D. (1998). Cell cycle arrest in Era GTPase mutants: a potential growth rate-regulated checkpoint in Escherichia coli. Mol Microbiol 27, 739-750.

Cashel, M., Gentry, D. R., Hernandez, V. J. \& Vinella, D. (1996). The stringent response. In Escherichia coli and Salmonella: Cellular and Molecular Biology, pp. 1458-1496. Edited by F. C. Neidhardt and others. Washington, DC: American Society for Microbiology.

Chen, X., Court, D. L. \& Xinhua, J. (1999). Crystal structure of ERA: a GTPase-dependent cell cycle regulator containing an RNA binding motif. Proc Natl Acad Sci U S A 96, 8396-8401.

Czyż, A., Jasiecki, J., Bogdan, A., Szpilewska, H. \& Wegrzyn, G. (2000a). Genetically modified Vibrio harveyi strains as potential bioindicators of mutagenic pollution of marine environments. Appl Environ Microbiol 66, 599-605.

Czyż, A., Wróbel, B. \& Węgrzyn, G. (2000b). Vibrio harveyi bioluminescence plays a role in stimulation of DNA repair. Microbiology 146, 283-288.

Freeman, J. A. \& Bassler, B. L. (1999a). A genetic analysis of the function of LuxO, a two-component response regulator involved in quorum sensing in Vibrio harveyi. Mol Microbiol 31, 665-677.

Freeman, J. A. \& Bassler, B. L. (1999b). Sequence and function of LuxU: a two-component phosphorelay protein that regulates a quorum sensing in Vibrio harveyi. J Bacteriol 181, 899-906.

Freeman, J. A., Lilley, B. N. \& Bassler, B. L. (2000). A genetic analysis of the functions of LuxN: a two-component hybrid sensor kinase that regulates quorum sensing in Vibrio harveyi. Mol Microbiol 35, 139-149.

Hause, B., Hause, G., Pechan, P. \& Van Lammeren, A. A. M. (1993). Cytoskeletal changes and induction of embryogenesis in microscope and pollen cultures of Brassica napus L. Cell Biol Int 17, 153-168.

Klein, G., Walczak, R., Krasnowska, E, Błaszczak, A. \& Lipińska, B. (1995). Characterization of heat-shock response of the marine bacterium Vibrio harveyi. Mol Microbiol 16, 801-811.

Klein, G., Żmijewski, M., Krzewska, J., Czeczatka, M. \& Lipińska, B. (1998). Cloning and characteriaztion of the dnaK heat shock operon of the marine bacterium Vibrio harveyi. Mol Gen Genet 259, 179-189.

Kok, J., Trach, K. A. \& Hoch, J. A. (1994). Effects on Bacillus subtilis of a conditional lethal mutation in the essential GTPbinding protein Obg. J Bacteriol 176, 7155-7160.

Lerner, C. G. \& Inouye, M. (1991). Pleiotropic changes resulting from depletion of Era, an essential GTP-binding protein in Escherichia coli. Mol Microbiol 5, 951-957.

MacKenzie, C., Chidambaram, C., Sodergren, E. J., Kaplan, S. \& Weinstock, G. M. (1995). DNA repair mutants of Rhodobacter spheroides. J Bacteriol 177, 3027-3035.

Meissner, P. S., Sisk, W. P. \& Berman, M. L. (1987). Bacteriophage $\lambda$ cloning system for the construction of directional cDNA libraries. Porc Natl Acad Sci US A 84, 4171-4175.

Okamoto, S. \& Ochi, K. (1998). An essential GTP-binding protein functions as a regulator for differentiation in Streptomyces coelicolor. Mol Microbiol 30, 107-119.

Quintarelli, G., Zito, R. \& Cifonelli, J. A. (1971). On phosphotungstic acid staining. I. J Histochem Cytochem 19, 641-647.

Sambrook, J., Fritsch, E. F. \& Maniatis, T. (1989). Molecular Cloning: a Laboratory Manual, 2nd edn. Cold Spring Harbor, NY : Cold Spring Harbor Laboratory.

Sayed, A., Matsuyama, S. \& Inouye, M. (1999). Era, an essential Escherichia coli small G-protein, binds to the $30 \mathrm{~S}$ ribosomal subunit. Biochem Biophys Res Commun 264, 51-54.

Scott, J. M. \& Haldenwang, W. G. (1999). Obg, an essential GTP binding protein of Bacillus subtilis, is necessary for stress activation of transcription factor $\sigma^{\mathrm{B}}$. J Bacteriol 181, 4653-4660. Sprang, S. R. (1997). G protein mechanisms: insights from structural analysis. Annu Rev Biochem 66, 639-678.

Spurr, A. R. (1969). A low-viscosity epoxy resin embedding medium for electron microscopy. J Ultrastruct Res 26, 31-43.

Swift, S., Throup, J., Bycroft, B., Williams, P. \& Stewart, G. (1998). Quorum sensing: bacterial cell-cell signaling from bioluminescence to patogenicity. In Molecular Microbiology, pp. 185-207. Edited by S. J. W. Busby, C. M. Thomas \& N. L. Brown. BerlinHeidelberg: Springer. 
Trach, K. \& Hoch, J. A. (1989). The Bacillus subtilis spoOB stage 0 sporulation operon encodes an essential GTP-binding protein. $J$ Bacteriol 171, 1362-1371.

Vidwans, S. J., Ireton, K. \& Grossman, A. D. (1995). Possible role for the essential GTP-binding protein Obg in regulating the initiation of sporulation in Bacillus subtilis. J Bacteriol 177, 3308-3311.

Wegrzyn, G. \& Taylor, K. (1992). Inheritance of the replication complex by one of two daughter copies during $\lambda$ plasmid replication in Escherichia coli. J Mol Biol 226, 681-688.
Węgrzyn, G., Kwaśnik, E. \& Taylor, K. (1991). Replication of $\lambda$ plasmid in amino acid-starved strains of Escherichia coli. Acta Biochim Pol 38, 181-186.

Yanisch-Perron, C., Vieira, J. \& Messing, J. (1985). Improved M13 phage cloning vectors and host strain: nucleotide sequence of the M13mp18 and pUC19 vectors. Gene 33, 103-119.

Received 15 May 2000; revised 11 September 2000; accepted 19 September 2000. 